\title{
Examining the ability of an FSO receiver to simultaneously communicate with multiple transmitters
}

\author{
Peter G. LoPresti*a, Shannon Suddath ${ }^{\mathrm{a}}$, Hazem Refai ${ }^{\mathrm{b}}$ Sarah A. Tedder ${ }^{\mathrm{c}}$, Bryan L. Schoenholz ${ }^{\mathrm{c}}$ \\ ${ }^{a}$ Department of Electrical Engineering, University of Tulsa, Tulsa, USA \\ ${ }^{b}$ Department of Electrical Engineering, University of Oklahoma, Tulsa, Tulsa, USA \\ ${ }^{\mathrm{c} N A S A}$ Glenn Research Center, Cleveland, Ohio, USA
}

\begin{abstract}
FSO-based communication systems experience difficulty with receiving and separating signals arising from multiple transmitters, a capability that would facilitate implementation of MIMO systems. Current implementations require multiple distinct optical antennas, each tracking a single user. This proves to be bulky and costly, especially if the transmitters are moving and must be tracked. A fiber-bundle receiver has the potential to use multiple pathways, corresponding to the individual fibers within the receiver, to capture different combinations of the incoming optical signals. If the bundle provides linear combining of the optical signals from both the individual fibers in the bundle and amongst the incoming optical signals, signal processing could extract the individual signals from the combinations. In this paper, we experimentally investigate whether the fiber-bundle receiver possesses sufficient linearity of operation to allow the separation of two signals by simple processing algorithms, for both turbulent and non-turbulent conditions. Data from two distinct sources enters a single-bundle, single field of view receiver, and a basis signal from one transmitter provides the reference for performing simple subtraction-based extraction of the unknown signal from the other transmitter. The experimental results show that the receiver does operate linearly, and that the linear operation remains sufficiently intact in the presence of turbulence to extract a recognizable copy of one signal from the other. The ability of the fiber bundle receiver to mitigate turbulence effects appears to assist in maintaining this sufficient level of linearity.
\end{abstract}

Keywords: Optical wireless, free-space optics, multiple transmitters, fiber-bundle receiver, turbulence

\section{INTRODUCTION}

As free-space optical (FSO) communication systems have continued to develop and become more capable, interest in the development of a variety of FSO-based networks has grown. In particular, recent work has focused on FSO systems that utilize one or more relay nodes to connect building-mounted transceiver nodes that do not possess direct line of sight. ${ }^{1}$ In such systems, several transmitters communicate with a relay node, and the relay node selects which transmitter to listen to with the help of algorithms that account for signal-to-noise ratio (SNR) and transmitter power to maximize the data throughput of the overall network. The concept can readily extend to other applications such as several aerial nodes communicating with wired networks through an access point, or the creation of airborne relay nodes connecting aerial transceivers over the horizon, with blocked line of sight due to weather, or to a set of ground stations.

A limiting factor for the development and expansion of relay-based FSO systems lies in the fact that current FSO receiver designs allow for the receiver to reliably communicate with only one transmitting node at a time. This requires the network to employ multiple receiving elements at the relay node equal to the number of current or projected transmitters. The restriction arises for two reasons. One, current FSO receivers possess a very narrow field of view (FOV) making it difficult for more than one signal to enter the receiver with sufficient strength to create a viable link. The receiver cannot therefore establish connections with a transmitter located at just one or two degrees from the optical axis of the receiver. Two, there exists only one path for the optical signal to reach the eventual optical-to-electrical conversion element. When two optical signals enter the receiver, only one composite electrical signal exits, which provides insufficient information for extracting or separating the two individual signals from the composite signal.

*peter-lopresti@utulsa.edu; phone 1918 631-3274; fax 1918 631-3344 
Due to these restrictions, the size, power requirements, and cost of the relay node scales upward with the number of network nodes served. For a relay node mounted on a building, cost would likely determine the maximum number of nodes served. For aerial or mobile relay nodes, size and power concerns would severely restrict the number of nodes served, as would the need to implement complex pointing, acquisition, and tracking systems to maintain strict alignment between nodes.

Receiver designs that possess large or expandable FOV and multiple optical paths can potentially make relay-based FSO networks viable in a wider range of applications. The fiber-bundle receiver design proposed and demonstrated by the investigators, and examined by other groups, ${ }^{2-5}$ possesses both of these desirable operating characteristics. The receiver design results in a base FOV of up to $\pm 7^{\circ}$, compared to standard designs with less than $\pm 0.5^{\circ}$. The very nature of the fiber bundle provides a number of pathways between the collecting optics and one or more optical-to-electrical converters, with the maximum equal to the number of fibers. The receiver can leverage these multiple pathways to communicate with multiple transmitters. Fig. 1(a) depicts a simple case with two optical-to-electrical converters, each receiving light from (for example) half of the fibers in the bundle, and Figure 1(b) depicts the experimental equivalent used in this work. If the transmitted signals enter the receiver in such a way that pathway one receives sufficient power from only transmitter one (Tx1) and the other pathway receives detectable power from both Tx1 and transmitter two (Tx2), the potential exists to use the signal from pathway one to reliably separate out Tx2's signal from that of Tx1 in pathway two, thereby allowing the receiver to establish connections with both Tx1 and Tx2 simultaneously. Algorithms adapted from RF communications may allow separation of the signals even if both pathways collect a detectable amount of optical power from both transmitters.

The experimental work reported in this paper investigates the ability of the receiver to linearly combine the signals from two transmitters without interference or other deleterious effects, especially in the presence of a turbulent channel. A receiver that performs linearly or nearly linearly under a range of operating conditions simplifies the process of separating and accurately reconstructing the individual optical signals. The investigation considers the receiver's operation as a function of angle between the transmitters, beam divergence of one transmitter, and the presence of turbulence. The experiments provide results for several different operating environments that help examine the effectiveness of the method.

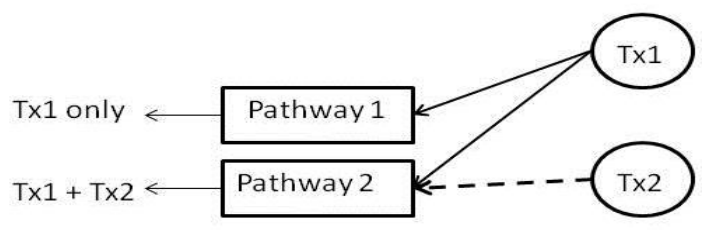

(a)

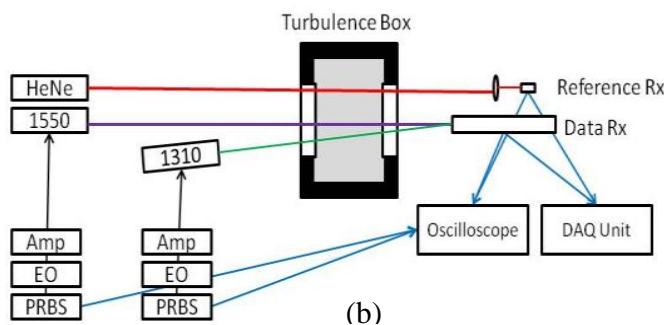

(b)

Figure 1. (a) Depiction of approach for receiving multiple signals with one receiver. (b) Experimental configuration.

\section{SYSTEM UNDER INVESTIGATION}

\subsection{Experimental Design}

Fig. 1(b) depicts the layout of the experimental system used to investigate the receiver. Two optical transmitters separated by an angle $\theta_{T x}$ direct data carrying optical beams towards a single fiber bundle-based optical receiver. Each transmitter consisted of an electrical-to-optical (EO) converter driven by a pseudo-random bit stream (PRBS) generator operating at a bit rate of $100 \mathrm{kbps}$. The maximum clock speed of the PRBS generators and the maximum sample rate of the data acquisition units prevented the testing of higher bit rates. The transmitter output coupled to an optical fiber, passed through an optical amplifier, and then coupled to an optical telescope for transmission through air. One EO converter operated at $1310 \mathrm{~nm}$ and the other operated at $1550 \mathrm{~nm}$. For a given experiment, Tx2, operating at $1550 \mathrm{~nm}$, remained at a fixed position and angle, while the position of Tx1, operating at $1310 \mathrm{~nm}$, varied such that the angle between the transmitted beams ranged between $4.47^{\circ}$ and $7.0^{\circ}$. A turbulence box, containing a heater and fans, generated different levels of turbulence along the path between the transmitters and receiver. A HeNe $(632.8 \mathrm{~nm})$ reference laser traveled parallel to the optical axis of the receiver and illuminated a separate detector. The reference laser provided data needed to estimate the value of the structure parameter $C_{n}{ }^{2}$ of the channel. 
The experiment uses the same optical receiver design reported in prior work. ${ }^{6}$ In short, a collecting lens array couples light to an array of large-core fibers. An optical system conditions and combines the light in the fibers and couples the light to a high-speed detector for conversion to an electrical signal. In the current work, graded-index lenses collimate the output light from the fibers instead of a lenslet array. Otherwise, all component details reported elsewhere remain unchanged.

A fast sampling oscilloscope and a data acquisition unit recorded data from the receiver, the reference detector, and the PRBS generators. The oscilloscope captured detailed (Gsamples/sec), short-term (2 ms) snapshots of the data, and the data acquisition unit captured coarse (Msamples/sec), long-term (2 minutes and longer) snapshots of the data. MATLAB scripts processed all of the collected data to determine $C_{n}^{2}$ of the channel, statistics of the receiver output, and measures of the quality of the separated signals.

\subsection{Experimental Procedure}

Each experiment conducted as part of the study followed the same procedure. At each choice of $\theta_{T x}$, the oscilloscope and data acquisition unit collected data at five different turbulence settings. The first four settings - no turbulence, low turbulence, moderate turbulence, and high turbulence - corresponded to the off, low, medium, and high settings of the heater within the turbulence box. After the high turbulence measurement, the lid of the box was removed, creating a highly turbulent flow during which the final readings of the experimental series were taken. Each instance of data collection consisted of six recordings of Tx1 transmitting only, Tx2 transmitting only, and both Tx1 and Tx2 transmitting. The recordings of Tx1 and Tx2 by themselves provided information about pathway 1 in Fig. 1(a) for this study, and to estimate the effective $C_{n}{ }^{2}$ (referred to as $C_{n e f f}{ }^{2}$ ) observed by the fiber-bundle receiver using the MATLAB scripts mentioned in the previous section. In this context, the effective value of $C_{n}^{2}$ refers to the effects of turbulence as experienced by the fiber-bundle receiver, as opposed to a standard receiver consisting of a single focusing lens coupled to an optical fiber. We expect that the structure of the fiber-bundle receiver will result in a lower variance in the received optical power compared to a traditional receiver, and thus the fiber-bundle receiver experiences the actual turbulence as if the turbulence was much lower - that is, $C_{n e f f}{ }^{2}<C_{n a c t u a l}{ }^{2}$. The experiments repeated the recording process for changes in $\theta_{T x}$ and the divergence of Tx2.

A beam profiler (Spiricon LT665-1550) placed in front of the fiber-bundle receiver provided data on the power distribution of the signal beam over time. The beam profiler utilizes a phosphor coated CCD camera in the NIR wavelengths (i.e., $1440 \mathrm{~nm}-1605 \mathrm{~nm}$ ) with an active area of $12.5 \mathrm{~mm} \times 10 \mathrm{~mm}$ and pixel pitch of $50 \mu \mathrm{m}$. The BeamGage analysis software controls the camera via a USB 3.0 PC interface and provides a graphical user interface for configuring beam measurements and performing statistical calculations. Beam profiler data processed through the analysis software provided an estimate of the $C_{n}^{2}$ value experienced by the signal beam.

Processing of the collected data proceeded as follows. The electrical signal driving Tx1 provided an example of the signal expected to arrive through pathway 1 in Fig. 1(a). The recording made with only Tx1 transmitting provided the scaling constant needed to convert the electrical signal into the effective signal obtained through pathway 1 . Therefore, the signal through pathway 1 was simulated according to

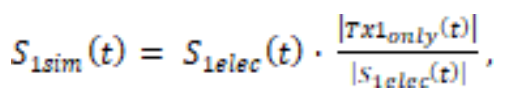

where $|f(t)|$ represents the peak-to-peak magnitude of the respective time-varying signal. The simulated signal was subtracted from the signal obtained through pathway 2 to obtain an estimate $\tilde{S}_{T \times 2}(t)$ of the signal from Tx2. The accuracy of the estimated signal was evaluated by comparing it to a scaled version of $S_{2 e l e c}(t)$ obtained using the process described

in (1). The comparison consisted of performing a correlation calculation on $\tilde{S}_{T \times 2}(t)$ and $S_{2 \text { elec }}(t)$ and calculating the average mean-squared error (MSE) between the two signals. A higher correlation and a lower average MSE corresponded to a more accurate reconstruction of the signal from Tx2. Recovery and comparison of the signal $\widetilde{S}_{T \times 1}(t)$ with $S_{\text {lelec }}(\mathrm{t})$ followed the same procedure.

\section{EXPERIMENTAL RESULTS}

\subsection{Assessing Linearity}

Figures 2 and 3 provide examples of the signals used in and obtained from processing of the receiver output for $\theta_{T x}$ of $5.25^{\circ}$ and $7.00^{\circ}$, respectively. The turbulence measured by the reference laser averaged a $C_{n}^{2}=7.8 \cdot 10^{-16} \mathrm{~m}^{-2 / 3}$ over six 
days of experiments. Figures 2(a) and 3(a) show the decrease in magnitude of the $1310 \mathrm{~nm}$ signal with increasing $\theta_{T x}$, and Figure 4 shows the ratio of the two magnitudes as a function of $\theta_{T x}$. As expected, the magnitude of the $1310 \mathrm{~nm}$ signal decreases with increasing angle, though a clearly defined $1310 \mathrm{~nm}$ signal remained visible for $\theta_{T x}$ up to $7.00^{\circ}$, which demonstrates the large inherent field of view of the receiver design. Given that the $1310 \mathrm{~nm}$ signal declines in magnitude relative to the $1550 \mathrm{~nm}$, the simple processing approach used here should likely produce better signal reproductions for the $1310 \mathrm{~nm}$ signal when the two signals have similar magnitudes and produce poorer reproductions of the $1310 \mathrm{~nm}$ signal for cases with highly disproportionate magnitudes. The comparisons of recovered to original signals presented in Figures 2 and 3 support this expectation. Note that the spiking observed at the beginning of each pulse in the $1550 \mathrm{~nm}$ bit stream - an artifact of the amplifier used - corrupts the reconstruction of the $1310 \mathrm{~nm}$ in all cases, especially for lower $1310 \mathrm{~nm}$ signal magnitudes. Attempts to filter the spike artifact degraded the bit transitions as well, corrupting the reconstruction process. Different methods for removing the artifact would likely improve reconstruction.

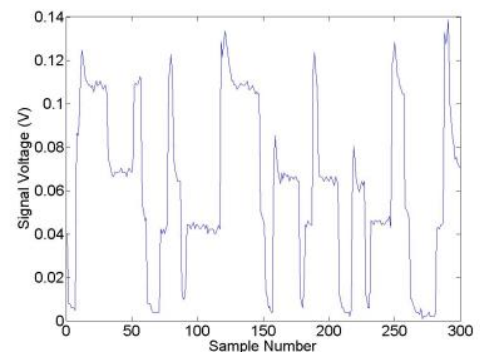

(a)

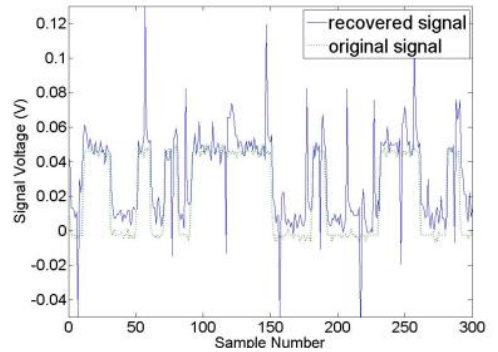

(b)

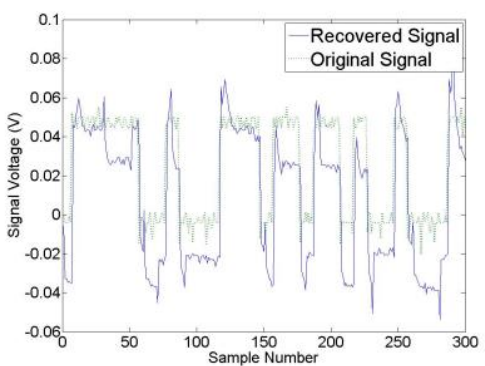

(c)

Figure 2. Example of collected and processed data for low turbulence at $\theta_{T x}=5.25^{\circ}$ (a) Output data from receiver, (b) comparison of transmitted and recovered $1310 \mathrm{~nm}$ signal, (c) comparison of transmitted and recovered $1550 \mathrm{~nm}$ signal.

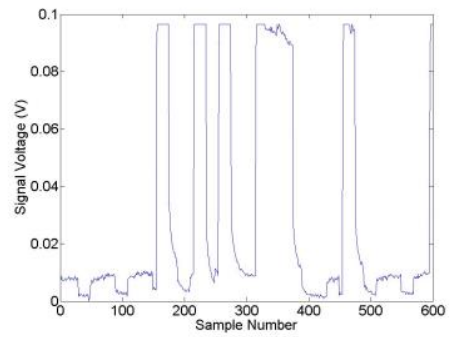

(a)

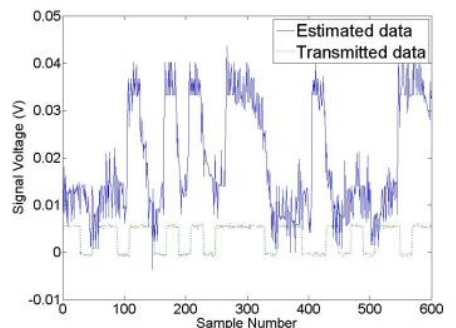

(b)

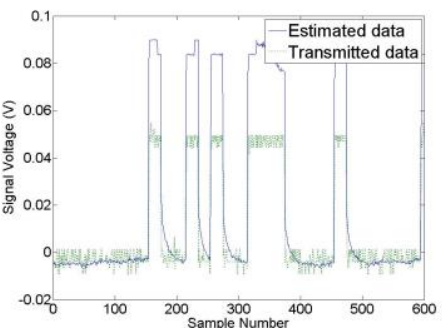

(c)

Figure 3. Example of collected and processed data for low turbulence at $\theta_{T x}=7.0^{\circ}$ (a) Output data from receiver, (b) comparison of transmitted and recovered $1310 \mathrm{~nm}$ signal, (c) comparison of transmitted and recovered $1550 \mathrm{~nm}$ signal.

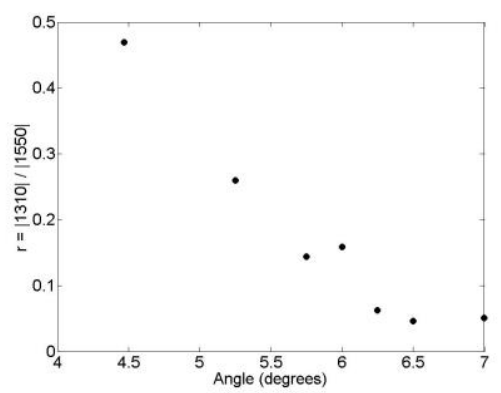

Figure 4. Ratio of $1310 \mathrm{~nm}$ to $1550 \mathrm{~nm}$ signal magnitude vs. angle.

Figures 5(a) and 5(b) show the dependence of the MSE and the normalized cross-correlation, respectively, on $\theta_{T x}$. The general trend over the angular range corresponds well with the observed signals in Figs. 3 and 4. The MSE for the 1310 $\mathrm{nm}$ varies significantly, depending on the size of the spiking artifact on the $1550 \mathrm{~nm}$ signal and the fidelity of reconstruction, while the $1550 \mathrm{~nm}$ MSE remains approximately constant once the $1310 \mathrm{~nm}$ magnitude falls below a 
value equal to 0.25 times the $1550 \mathrm{~nm}$ magnitude. The cross-correlation dependence on angle reinforces the decrease in the fidelity of the recovered $1310 \mathrm{~nm}$ signal as the mismatch in signal magnitude increases.

In all cases tested, the receiver displayed sufficient linearity to allow a simple linear algorithm to provide an estimate of both signals incident on the receiver. For cases where the ratio of the signal magnitudes is large, higher fidelity reconstruction will require a more sophisticated processing algorithm than the one used here. The next step consists of evaluating whether the linearity and the dependencies indicated in Figure 5 remain consistent with increased beam divergence and increasing turbulence along the transmission path.

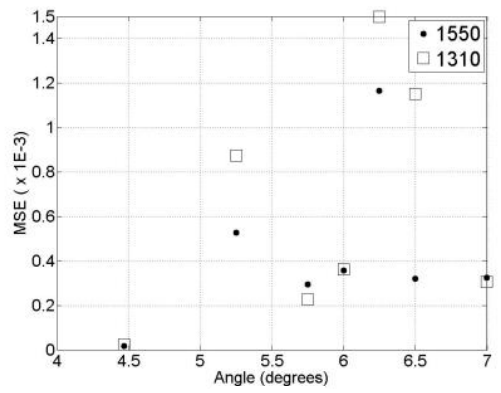

(a)

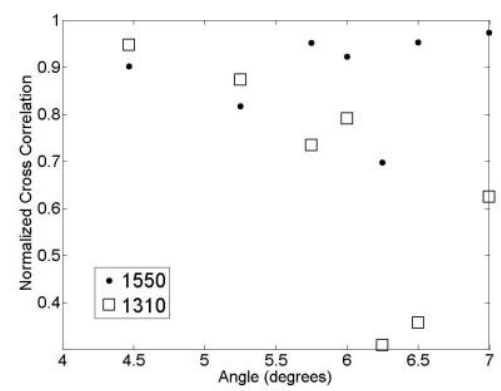

(b)

Figure 5. Dependence of (a) MSE and (b) normalized cross correlation on angle and wavelength.

\subsection{Dependence on Divergence and Turbulence}

Varying the optics of the telescope on Tx2 allowed the divergence of the $1550 \mathrm{~nm}$ beam to vary. The beam size at the receiver varies from approximately the size of one lens in the input lens array (nearly collimated, or coupled mainly to one fiber in the bundle) to overfilling the input aperture of the collecting bundle of fibers $(1 \mathrm{~cm}$ in diameter). The divergence of Tx1 $(1310 \mathrm{~nm})$ did not vary due to the already small signal magnitude available at the larger $\theta_{T x}$. Initial experiments used the same turbulence level as previously noted.

Fig. 6(a) shows the relationship obtained between the normalized cross correlation of the recovered signal with the original signal for both $1310 \mathrm{~nm}$ and $1550 \mathrm{~nm}$, and the increase in beam width of the $1550 \mathrm{~nm}$ signal due to increased divergence. Fig. 6(b) shows the corresponding ratio between magnitudes of the received signals. For beam widths up to and including $1.1 \mathrm{~cm}$, the signal ratio displays a quadratic dependence on beam width, which follows from the quadratic decrease in peak optical power of the $1550 \mathrm{~nm}$ signal with increasing divergence. The cross correlation dependence on beam width reflects the change in beam ratio and peak $1550 \mathrm{~nm}$ signal, with the accuracy of $1310 \mathrm{~nm}$ signal recovery increasing and the accuracy of $1550 \mathrm{~nm}$ signal recovery decreasing. However, as the $1550 \mathrm{~nm}$ beam divergence increases further, the received signal amplitude increases as lens-fiber combinations away from the central axis of the receiver intercept and capture transmitted optical power that diverged away from the central axis. Instead of the signal reconstruction continuing to degrade as the divergence increases, the reconstruction improves and remains of higher quality, at least until a significant fraction (in this case $w_{\text {beam }} / w_{R x}=2.8$ ) of the transmitted power falls outside of the input aperture of the lens array. This behavior should improve the range of divergence for successful transmission, and hints at the mechanism by which the receiver can increase overall link performance in the presence of turbulence.

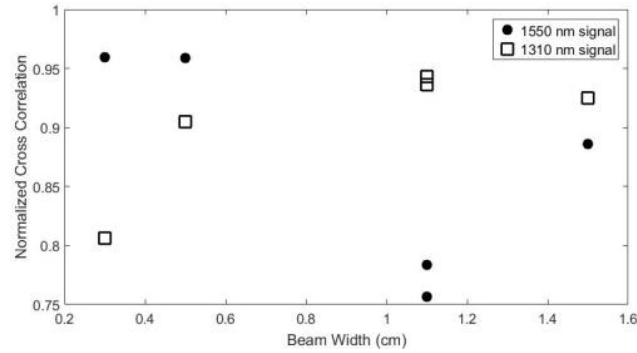

(a)

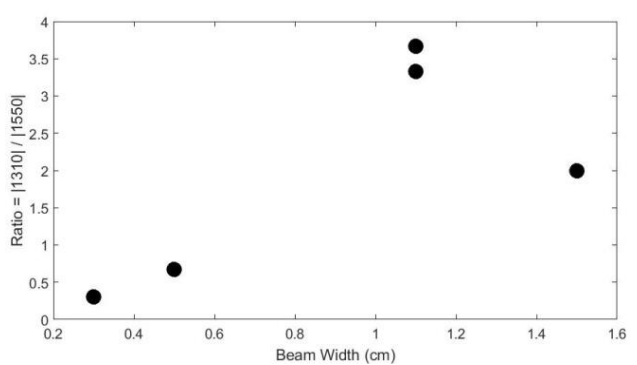

(b)

Figure 6. (a) Normalized cross correlation and (b) Ratio of the received signal magnitude, $|1310 \mathrm{~nm} /||1550 \mathrm{~nm}|$, as a function of the beam width of the $1550 \mathrm{~nm}$ beam. 
Figure 7 shows the performance of the link in the presence of different levels of turbulence between the transmitters and the receiver. The plots include the calculated cross correlations for every collection event to indicate the variation observed for each angle and each turbulence condition. For reference, Figure 5(b) shows the averaged results of the data in Figure 7(a). The corresponding structure constants, as measured by the reference laser, were $C_{n}{ }^{2}=7.8^{\cdot} \cdot 10^{-16} \mathrm{~m}^{-2 / 3}$ for low turbulence, $C_{n}{ }^{2}=4.6 \cdot 10^{-15} \mathrm{~m}^{-2 / 3}$ for medium turbulence and $C_{n}{ }^{2}=2.1 \cdot 10^{-14} \mathrm{~m}^{-2 / 3}$ for the high turbulence experiment. Two significant trends emerge from the data that shed light on the operation of the receiver. First, the average value of the normalized cross correlation for the $1310 \mathrm{~nm}$ reconstruction decreased as turbulence increased, particularly at the lower angles. Second, as shown in Figure 8, the standard deviation of the measured cross correlations did not vary systematically with the amount of turbulence present in the link. Although the standard deviation slowly increased in general with increasing angle, the turbulence condition with the lowest standard deviation and the turbulence condition with the highest standard deviation did not remain consistent over the range of angles, with the high turbulence condition sometimes producing the lowest variation in the standard deviation. Thus, despite variations in the power distribution of the transmitted beam induced by the turbulence, the quality of the reconstruction depended more significantly on the angle between the transmitters - the standard deviation increases slightly with increasing angle in Figure 8 - which here determines primarily the relative collected signal strength of the transmitted beams at the receiver.

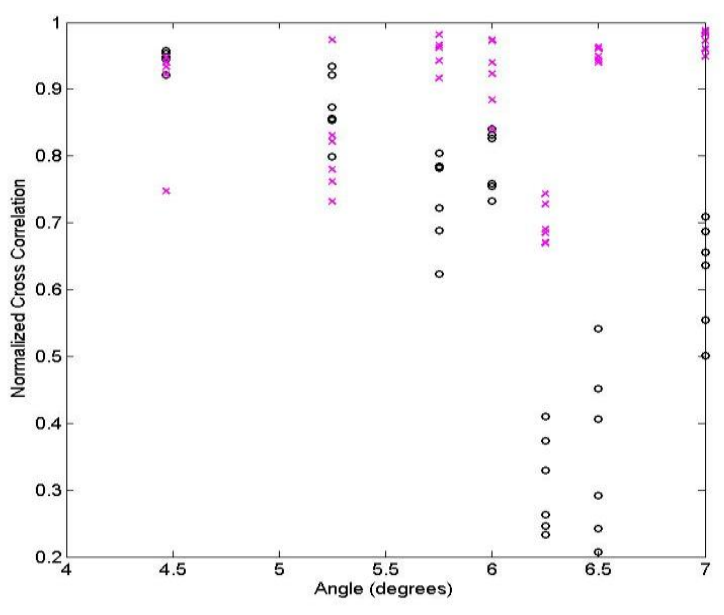

(a)

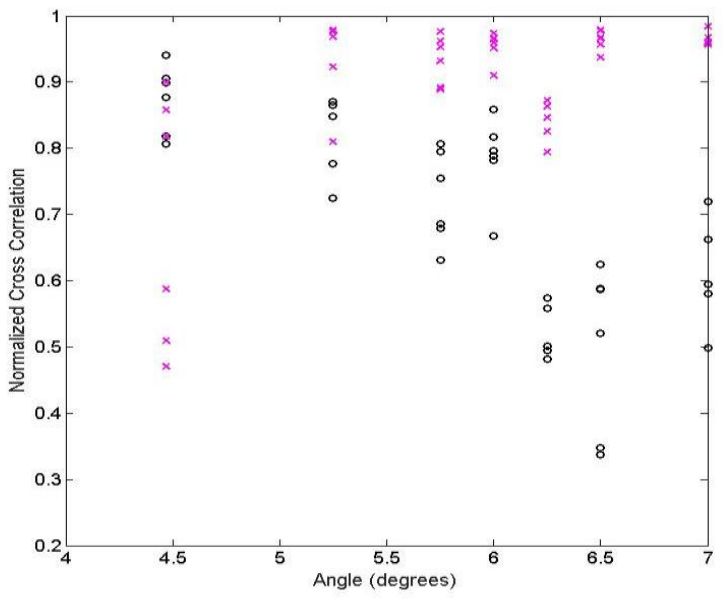

(b)

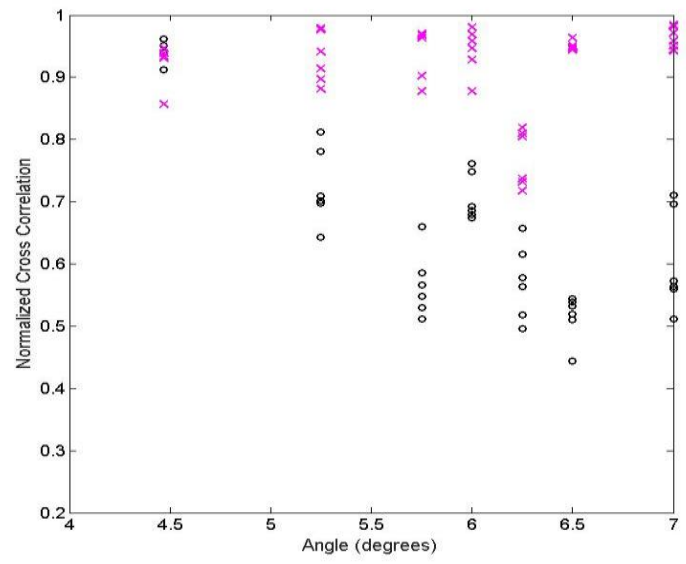

(c)

Figure 7: Normalized cross correlation variation as a function of angle and wavelength under (a) low turbulence conditions, (b) medium turbulence conditions, and (c) high turbulence conditions. $\mathrm{X}=$ reconstruct stronger signal knowing weaker signal. $\mathrm{O}=$ reconstruct weaker signal knowing stronger signal. 
To investigate the lack of dependence further, additional processing of the data compared the variations observed in the "1" data collected by the fiber-bundle receiver to the variations in the incoming infrared signal beam. The processing program collected all signal data associated with a transmitted " 1 ", accounted for electrical noise in the detector, and provided an estimate of the $C_{n}{ }^{2}$ value. The $C_{n}{ }^{2}$ value so calculated represents the effective impact of turbulence as experienced by the receiver, influenced by the collecting abilities of the lens-fiber array. Figure 9 shows a typical relationship between the measured $C_{n}{ }^{2}$ value for the incoming signal beam and the effective $C_{n}{ }^{2}$ value experienced by the receiver. In all experimental cases, the effective $C_{n}{ }^{2}$ value experienced by the receiver initially increases and then begins to decrease somewhat as the actual turbulence in the turbulence box increases. Observations of the incoming signal beam's power distribution using the beam profiler verified that no other aspects, other than the turbulence, of the experimental setup altered the power distribution of the beam.

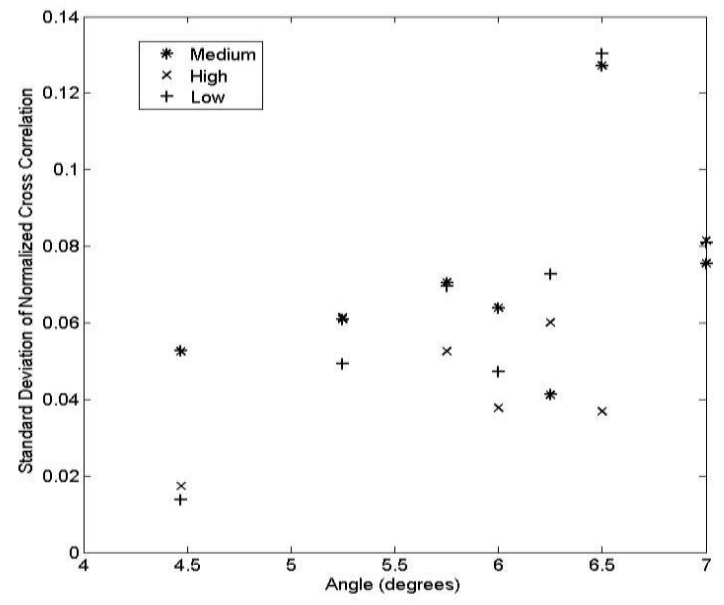

Figure 8: Standard deviation of the normalized cross correlation for $1310 \mathrm{~nm}$ signal as a function of angle under varying turbulence conditions.

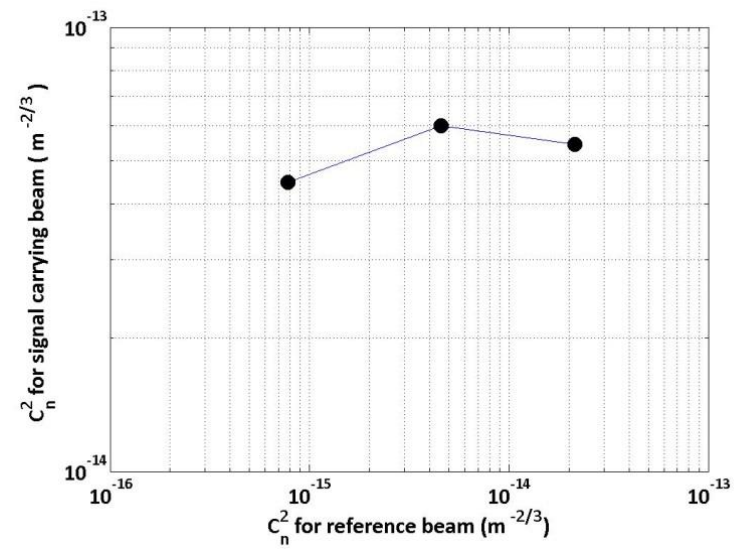

Figure 9: Calculated $C_{n}{ }^{2}$ value for the signal beam as a function of the measured $C_{n}{ }^{2}$ value for the incoming signal beam.

\section{CONCLUSION}

We have demonstrated a fiber-bundle design for FSO communication that possesses the characteristics necessary to facilitate simultaneous communication with two independent optical sources of data. Simple algorithms using knowledge of the original transmitted data allowed reliable separation of the data streams, even for transmission across turbulent channels. We expect that more sophisticated approaches, such as independent component analysis, combined with the characteristics of the receiver design, will allow separation of data streams even without knowledge of the original data transmitted from either source. 
The fiber-bundle receiver design also demonstrates reduced sensitivity to the effects of turbulence compared to that of traditional receiver designs. The lens-fiber bundle design of the receiver likely contributes to the observed dependence of the effective $C_{n}{ }^{2}$ value on the actual turbulence strength in the following manner. For weaker turbulence, small deflections occur in the signal beam, causing more of the transmitted power to fall on the gaps between adjacent lenses in the bundle, effectively increasing fading in the collected optical power. As the turbulence strength increases and deflects the power in the transmitted beam over a larger angle, the deflected power falls within the acceptance cone of the lens-fiber pairs adjacent to the central pairs, and these adjacent lens-fiber pairs collect optical power from the signal despite the deflections caused by turbulence. The array of lens-fiber pairs can therefore collect more of the signal power compared to the operation of traditional, single lens-pair receivers, reducing the magnitude of signal power fades experienced under more turbulent conditions.

\section{REFERENCES}

[1] A. N. Kopeikin, S. S. Ponda, L. B. Johnson, O. Toupet, and J. P. How. "Real-time dynamic planning to maintain network connectivity in a team of unmanned air vehicles." Proc. IEEE Global Communication Workshop (Wi-UAV 2011), Houston, TX, 2011.

[2] D. V. Hahn, D. M. Brown, N. W. Rolander, J. E. Sluz, and R. Venkat, "Fiber optic bundle array wide field-of-view optical receiver for free space optical communications," Optics Letters, 35(21), pp. 3559-3561, 2010.

[3] A. M. Brown, D. V. Hahn, D. M. Brown, N. W. Rolander, C.-H. Bair, J. E. Sluz, "Experimental implementation of fiber optic bundle array wide FOV free space optical communications receiver," Applied Optics, 51(18), pp. 3995 4002, 2012.

[4] K. Wita, S. Hejduk, V. Vasinek, J. Bitasek, J. Latal, "Low noise omnidirectional optical receiver for the mobile FSO networks," Proc. SPIE, vol. 8774, pp. 87741Q-1 - 87741Q-12, 2013.

[5] D. Zhou, P. G. LoPresti, H. Refai, "Design analysis of a fiber-bundle-based mobile free-space optical link with wavelength diversity," Applied Optics, vol. 52, no. 16, pp.3689-3697, 2013.

[6] P. LoPresti, N. Hutchins, S. Kohrmann, M.F. Babelli, H. H. Refai, "Wavelength dependence of a fiber-bundle based FSO link,” Globecom Workshops, pp. 493-498, 2014. 